\title{
A new computer mouse called Horse
}

\author{
C.J. Snijders and P.C. Helder, \\ Erasmus MC, University Medical Center Rotterdam, \\ the Netherlands.
}

Tn the early 60's Doug Engelbart, a scientist at the Stanford 1 Research Institute in California, invented the computer mouse. This may certainly be seen as one of those brilliant tools we hardly can do without. However excellent the functionality of the mouse-we all use it with great ease-its recent form can also be a significant source of discomfort.

Repetitive movements cause physical complaints. This is one of the causative factors of Repetitive Strain Injury (RSI) or Occupational Overuse Syndrome (OOS) [1]. Rigid work patterns and prolonged periods of heavy work load can result in physical stress. Personality aspects are also considered to play a role in the development of RSI [2].

About 20\% of the working population has complaints related to the neck-shoulder-arm region and overuse complaints can be the result of using the mouse. This is why a biomechanical research program was started by Erasmus MC, University Medical Center Rotterdam, the Netherlands.

\section{Biomechanical model}

The present study has identified one cause for several physical problems in the neck-shoulder-arm region.

Based on a pilot study a hypothesis was introduced that forceful gripping or pinching goes with tension in the deep neck muscles. To investigate this, blood flow velocity was measured in the region indicated in Fig. 1 (with permission from Sobotta, Atlas of Human Anatomy).

The drawing shows the costoclavicular gate between clavicular bone and first rib (see circle).

Neck muscle tension results in a narrowing of this gate and thus impingement of the subclavian artery and vein.

This conclusion is supported by a biomechanical model of the aetiology of tennis elbow [3]. It describes the role of extensor muscles in the hand and lower limb and substantiates the finding that restriction of blood flow is caused by forceful pinching. Therefore we conclude that certain force application by the hand is related to tension in the deep neck muscles which explains a variety of shoulder- and arm complaints.

\section{Design of a computer mouse called "Horse"}

The use of a conventional computer mouse requires continuous lifting of the fingers. This results in excessive use of extensor muscles to avoid unwanted switching which can be seen as a possible cause of tension in the deep neck muscles. It was therefore decided to design a mouse that does not provoke extensor muscle activity.

This new concept is called Horse in view of its functional design: the palm of the hand as well as the middle three fingers "sit" on the main body while thumb and little finger rest at a lower level at the side as if supported by stirrups (Fig. 2). Thumb and little finger work together to realize optimal control in the horizontal (X-Y) plane. The design of the Horse allows for the

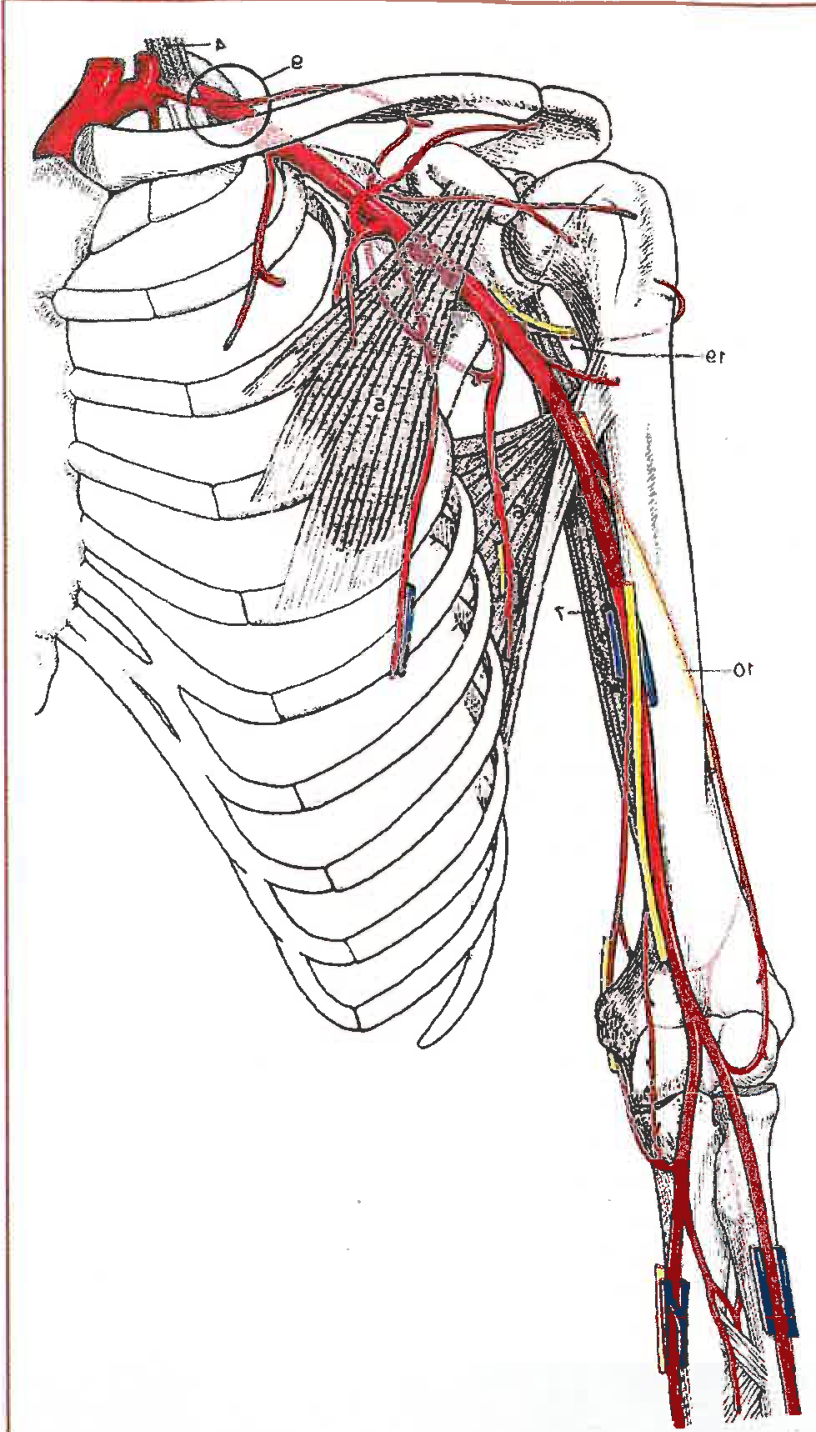

- Fig. 1: Narrowing of costoclavicular gate (see circle) resulting in impingement of artery and vein (with permission from Sobotta, Atlas of Human Anatomy)

three middle fingers to adopt a flexed position to relax the tendons. A major part of these fingers rests in a more or less vertical position.

In view of this supported position of the hand and fingers, extensor muscles can relax.

Extensor action is no longer required with the palm of the hand and fingers resting on the Horse. A light flexing action of the top of the fingers is sufficient to switch. This action results in a force on the touch switches situated at the lower end near the tips of the fingers.

These switches react on touch force only which reduces or virtually eliminates the movement of the tendons when switching. Moreover, complete support of the fingers will reduce the necessity of co-contraction of the intrinsic muscles of the hand [4]. Other features as a result of the design of the Horse are:

- a better stability control in the X-Y plane

- the possibility to reduce friction of the Horse on the table top 


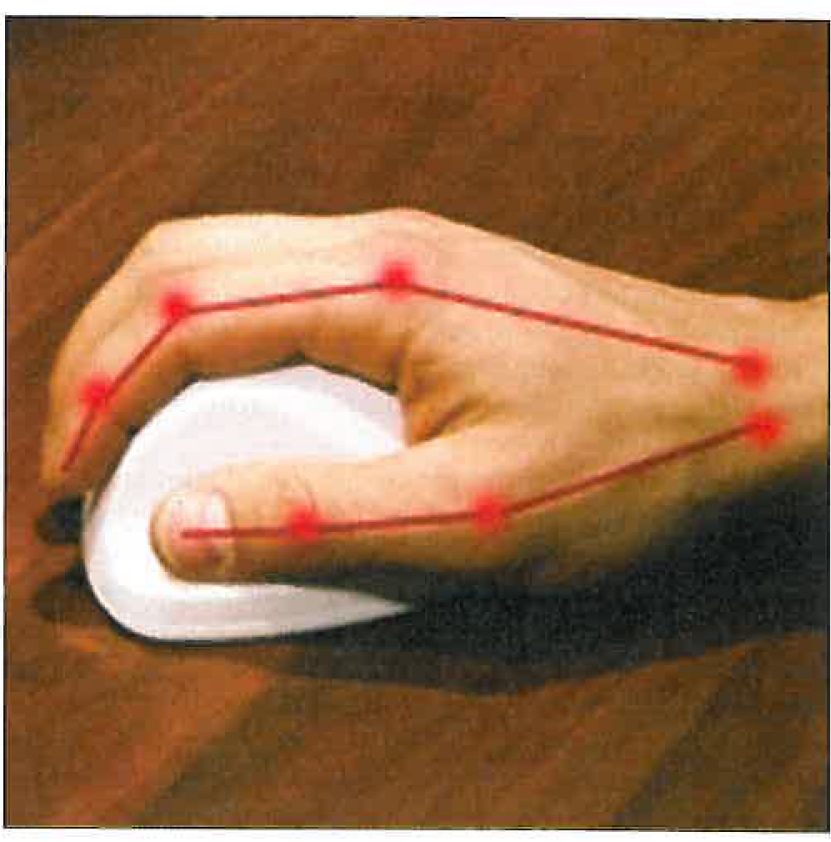

A Fig. 2: Thumb at the side, major part of the three fingers more or less vertical, finger tips touch at the side of the switches.

\section{Result of development}

A working concept of the Horse was produced using Rapid Proto Typing (RPT). This technology generates prototype bodies directly from 3D Computer Aided Design (CAD) files.

The electronics of a conventional mouse were installed in the RPT body. The prototype was then tested in a day-to-day working environment. Horse and mouse could thus be compared.

Figure 3 shows a hand on the Horse manufactured by means of RPT and on a conventional mouse. Relevant joints, phalanges and transversal contours are indicated in red clearly showing the difference between the two.

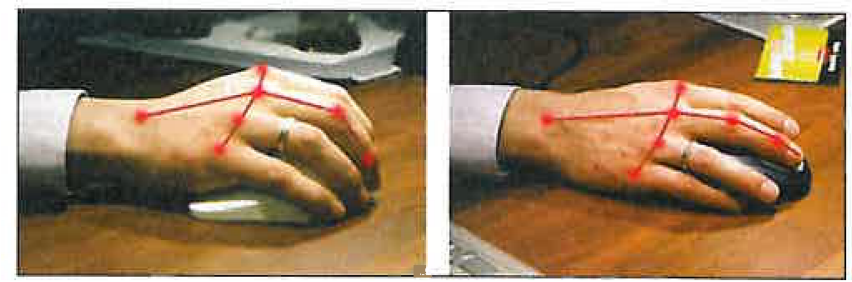

\section{Fig. 3: RPT Horse, relaxed hand; conventional mouse,} stressed hand.

\section{Production}

A working concept of the Horse was produced using Rapid Proto Typing (RPT). This technology generates prototype bodies directly from 3D Computer Aided Design (CAD) files.

The electronics of a conventional mouse were installed in the RPT body. The prototype was then tested in a day-to-day working environment. Horse and mouse could thus be compared.

Figure 3 shows a hand on the Horse manufactured by means of RPT and on a conventional mouse. Relevant joints, phalanges and transversal contours are indicated in red clearly showing the difference between the two.

\section{Conclusion}

The combination of fundamental research based on the anatomy of the human body and the availability of state of the art technology, enables the production of small series of custom made products prior to large-scale production. In this case the development and production of a computer mouse which aims to provide an appropriate extension of the human body.

The selected approach aims at reducing the risks involved with market introduction of new products.

\section{About the authors}

Chris J. Snijders, PhD, Erasmus MC, University Medical Center Rotterdam

Research on biomechanics of low back and pelvic pain and on neck-, shoulder- and arm-problems. Product design for a number of medical disciplines.

Teaching: undergraduate and graduate courses for medical and engineering students in theoretical mechanics, biomechanics and product design.

Paul C. Helder, MBA, BA, BSc

Management of innovation, as member of the board of commercial organizations involved in various developments that combine mechanical engineering and biotechnology.

\section{References}

[1] Yassi A. Repetitive strain injuries. Lancet 1997; 349: 943-947

[2] Health Council of the Netherlands: RSI. The Hague: Health Council of the Netherlands, 2000; publication nr. 2000/22

[3] Snijders C.J., Volkers A.C.W., Mechelse K., Vleeming A. Provocation of epicondylalgia lateralis (tennis elbow) by power grip or pinching. Medicine and Science in Sports and Exercise 1987; 19(5): 518523

[4] Leijnse J.N.A.L., Bonte J.E., Landsmeer J.M.E., Kalker J.J., Meulen J.C. van der, Snijders C.J. Biomechanics of the finger with anatomical restrictions; the significance for the exercising hand of the musician. Journal of Biomechanics 1992; 25(11): 1253-1264

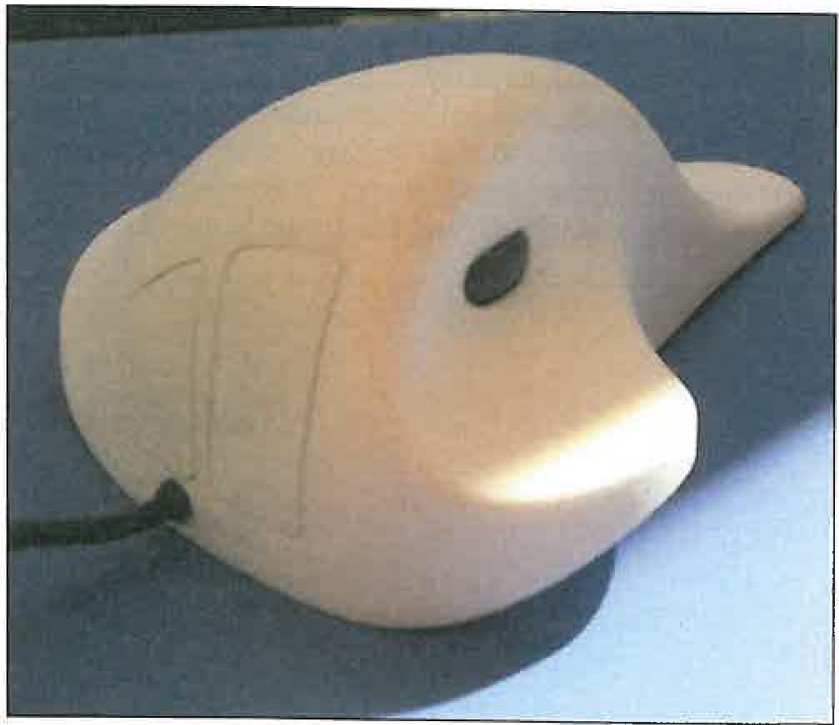

4 Fig. 4: Front view of the Horse manufactured by means of RPT with touch switches and scrool wheel. 